\title{
Estratégias para reorganização da Rede de Atenção à Saúde em resposta à Pandemia COVID-19: a experiência do Sistema de Saúde Italiano na região de Lazio $^{1}$
}

Renato Tasca, Adriano Massuda

\section{RESUMO}

O presente artigo faz uma breve síntese de estratégias adotadas pelos gestores de saúde da rede pública da região de Lazio-IT a partir de informações coletadas em entrevistas com informantes-chave, atualizadas em torno de um mês após a notificação do primeiro caso na Itália. $O$ estudo aponta ques as estratégias adotadas pela região seguiram um plano de enfretamento da pandemia de definido de acordo com cenários epidemiológicos. Acredita-se a análise da experiência italiana pode ser de grande valia para identificar erros a serem evitados e caminhos que podem ser seguidos em outros países na resposta a COVID-19. A análise da experiência em curso também serve de reflexão a respeito dos enormes desafios, presentes e futuros, para a saúde global, em particular para a organização de sistemas e serviços, frente a uma grave situação de emergência em Saúde Pública, como apresentada pela COVID-19.

Palavras-chave: Pandemia; COVID-19; Atenção Primária à Saúde.

Revista da Rede APS 2020

Publicada em: 15/04/2020

DOI:10.14295/aps.v2i1.65

Renato Tasca

(Organização Pan Americana da Saúde/OPAS, Brasil);

Adriano Massuda

(FGV-EAESP, Brasil)

Correspondência para:

Renato Tasca

tascar@paho.org

\section{ABSTRACT}

This article provides a brief summary of strategies adopted by public health managers in the Italian region of Lazio. The study shows that the strategies adopted by the region were changed to match the epidemiological scenarios. Such an analysis is believed to be of great value in identifying paths that can be followed by other countries in the response to COVID-19. The analysis of the ongoing experience also serves as a reflection on the enormous challenges, present and future, for global health, in particular for the organization of systems and services, in the face of a serious emergency situation in Public Health, as presented by COVID -19.

Keywords: Pandemic; COVID-19; Primary Health Care. 


\section{INTRODUÇÃO}

A Itália é um dos países europeus mais afetados pela pandemia provocada pelo coronavírus 2019 (COVID-19). Desde 21 de fevereiro de 2020, quando o primeiro caso da COVID-19 foi registrado no país, o Serviço Nacional de Saúde, que oferece acesso universal à saúde, tem operado próximo do colapso nas regiões mais afetadas do país, como a Lombardia ${ }^{2}$. Diante do agravamento da situação da epidemia, desde o 13 de março 2020 toda a população residente no território italiano está sujeita a quarentena.

A dramática experiência vivida nas áreas que foram primeiramente afetadas na Itália abriu uma janela de tempo para que as demais regiões do país pudessem adotar estratégias de contenção da propagação do vírus e organização do sistema de saúde para enfrentar a epidemia. Dentre elas, a Região de Lazio, que abrange a cidade de Roma, implementou um conjunto de medidas para reorganizar fluxos assistências de acesso e atendimento no sistema de saúde para pacientes COVID e não-COVID, de acordo com as diferentes fases de evolução da epidemia.

Nesse artigo, apresentamos uma breve síntese de estratégias adotadas pelos gestores de saúde da rede pública da região de Lazio. As informações foram coletadas a partir de entrevistas com informantes-chave, entre os quais gestores e pesquisadores italianos, e consulta a documentos oficiais, incluindo portarias emanadas pela Secretaria de Saúde da região. As informações foram atualizadas ao dia 26/03/2020 - pouco mais de um mês depois da notificação do primeiro caso no território nacional.

A análise da experiência italiana pode ser de grande valia para identificar erros a serem evitados e caminhos que podem ser seguidos em outros países na resposta a COVID-19. Entretanto, mais do que receitas para outros países, a análise da experiência em curso serve de reflexão a respeito dos enormes desafios, presentes e futuros, para a saúde global, em particular para a organização de sistemas e serviços, frente a uma grave situação de
Emergência em Saúde Pública, como apresentada pela COVID-19.

\section{O SISTEMA DE SAÚDE NA REGIÃO DE}

\section{LAZIO E A RESPOSTA À PANDEMIA}

Brasil e Itália possuem sistemas universais de saúde, porém com mecanismos de gestão e governança bastante diferentes. $\mathrm{Na}$ Itália, o Ministério da Saúde e os municípios não têm atuação direta na organização da atenção à saúde. Essa responsabilidade é atribuição da região - o que correspondente aos estados no Brasil. A plena governança da Região sobre a rede de saúde foi um fator determinante no planejamento e na definição de medidas de reorganização dos serviços apresentadas neste material.

A Região de Lazio tem uma população um pouco inferior a seis milhões de habitantes (10\% da população do país), sendo que metade vive na capital, Roma. Os serviços de maior complexidade são concentrados na capital, mas existe uma boa rede de serviços de saúde distribuída em todo o território regional.

Com 3.264 casos confirmados da COVID-19 em 31 de março de 2020, esta Região ocupava o sétimo lugar no ranking das regiões mais afetadas. Até 09 de abril de 2020, os serviços de saúde da região não vivenciaram situações caóticas como as da Lombardia, que registrou mais de 40 mil casos da COVID-19 no mesmo período.

As estratégias adotadas pela região seguiram um plano de enfretamento da pandemia de definido de acordo com cenários epidemiológicos, conforme fases sistematizadas e descritas a seguir:

\section{I) FASE 01: ESTRATÉGIAS PARA REORGANIZAR O ACESSO E OS PAPEIS DE SERVIÇOS NÃO- HOSPITALARES}

As primeiras medidas foram desencadeadas quando foram registrados os primeiros casos da COVID-19 na região (figura 1 ): 


\section{I.1) Porta de entrada - acesso telefônico e presencial.}

Com o intuito de limitar ao mínimo o acesso espontâneo e presencial aos prontos atendimentos e emergências de hospitais, o primeiro acesso aos serviços de saúde passou a realizado por via telefônica.

Apenas em casos mais graves ou urgentes as pessoas se dirigem diretamente os serviços de saúde. Nesses casos, normalmente os pacientes são recebidos em estruturas externas as unidades, frequentemente tendas acondicionadas e equipadas de forma adequada (ver depois o fluxo hospitalar). Só entra na unidade hospitalar quem precisar de internação.

\section{I.2) 0800 para Informações e Teleconsulta.}

Um serviço telefônico 0800 foi criado para prestar informações gerais sobre COVID-19 e atender os casos com sintomas respiratórios "leves". Na grande maioria dos casos, a consulta telefônica é suficiente para responder a demanda dos pacientes e para ativar os serviços de vigilância remota, em caso de necessidade. Em menor número, o serviço telefônico também ativa a unidade de assistência domiciliar. As principais categorias de demandas atendidas pelo 0800 são:

- Pessoas de grupos de risco, em aparente estado de boa saúde, mas impossibilitados a garantir a ausência de contatos com pessoas afetadas pelo COVID-19 no próprio domicílio, que solicitam internação num centro de acolhimento (ver depois)

- Pessoas que entraram em contato com indivíduos positivos ou suspeitos de doença COVID-19, para receber orientações sobre isolamento; se for o caso, é ativado o sistema de vigilância remota.

-Indivíduos com sintomas suspeitos de COVID19, ainda leves, para definir conduta diagnostico-terapêutica a ser decidida em cada caso e ativar a vigilância remota, quando necessário.
-Pessoas com sintomatologia leve, positivos para COVID-19 para os quais, após avaliação das condições clínicas do paciente e a situação logística / de moradia, foi indicado isolamento em casa ou outro lugar adequado, com vigilância remota.

- Pacientes que receberam alta hospitalar clinicamente curados, ainda com COVID-19 positivo, que precisam isolamento e vigilância remota.

\section{I.3) Serviços de Atenção Primária em Saúde (APS).}

A APS tem papel central na resposta à COVID-19. Entretanto, foi preciso modificar o modo de funcionamento durante o período da epidemia. Os médicos de família não realizam o primeiro atendimento clínico de pacientes suspeitos ou infectados pela COVID-19. Por outro lado, estes profissionais colaboram ativamente na vigilância remota desses pacientes com quadros assintomáticos, convalescentes ou com sintomas leves.

Além disso, a medicina de família mantém, em via remota a atenção aos pacientes para atividades de rotina e de acompanhamento de pessoas em risco (crónicos, materno-infantil, etc.). Os atendimentos são feitos por meio do uso de telefone ou tele-atendimento para evitar consultas presenciais. As vistas domiciliares também são limitadas aos pacientes não-COVID.

\section{I.4) Serviços de Vigilância domiciliar remota.}

Os serviços de vigilância domiciliar remota destinam-se a aos casos positivos e aos que estiveram em contato com positivos. Pode ser ativada tanto pelo telefone ao 0800 Teleconsulta, por aplicativo, ou pelo serviço de ambulância. $\mathrm{Na}$ presença de condições socioambientais que não garantem um isolamento seguro no domicílio, a pessoa é encaminhada para instalações residenciais destinadas à fase de avaliação ou quarentena.

I.5) Serviços de atendimento virtual: Website e aplicativo. 
Tasca, R.; Massuda, A.

Os usuários têm acesso a uma ferramenta web de Teleconsulta no site da Região de Lazio, acessível também via aplicativo para smartphone intitulada Lazio Doctor per Covid, com suporte vídeo e envio documentos e fotos. Além de vários serviços informativos, o instrumento proporciona acesso ao questionário de autoavaliação e permite ativar a vigilância remota nos casos previstos.

\section{I.6) Serviços de acolhimento a grupos vulneráveis: "Struttura Protetta"}

As pessoas positivas ou suspeitas que precisam ficar em isolamento, assintomáticas ou com sintomatologia leve, mas que não têm condições adequadas para permanecer com segurança no próprio domicílio, são transferidas em centros de acolhimento, onde serão acompanhados pelo sistema de vigilância remota.

\section{I.7) Serviços pré-hospitalar móvel - Ambulância - 112/118 [SAMU da Itália]}

Os profissionais do 118 realizam entrevista/triagem por telefone. Respondem as chamadas de pessoas com sintomas clínicos importantes - febre, tosse, dificuldades respiratórias ou condições de emergência. 0 objetivo da entrevista é identificar se a pessoa deverá ser encaminhada (com transporte seguro, proporcionado unicamente pelo SAMU) ao hospital de referência (definido pela central de regulação da Região Lazio), ou se deverá ficar em casa sob vigilância.

Figura 1 - Fluxo da pessoa com sintomatologia gripal ou contato de positivo

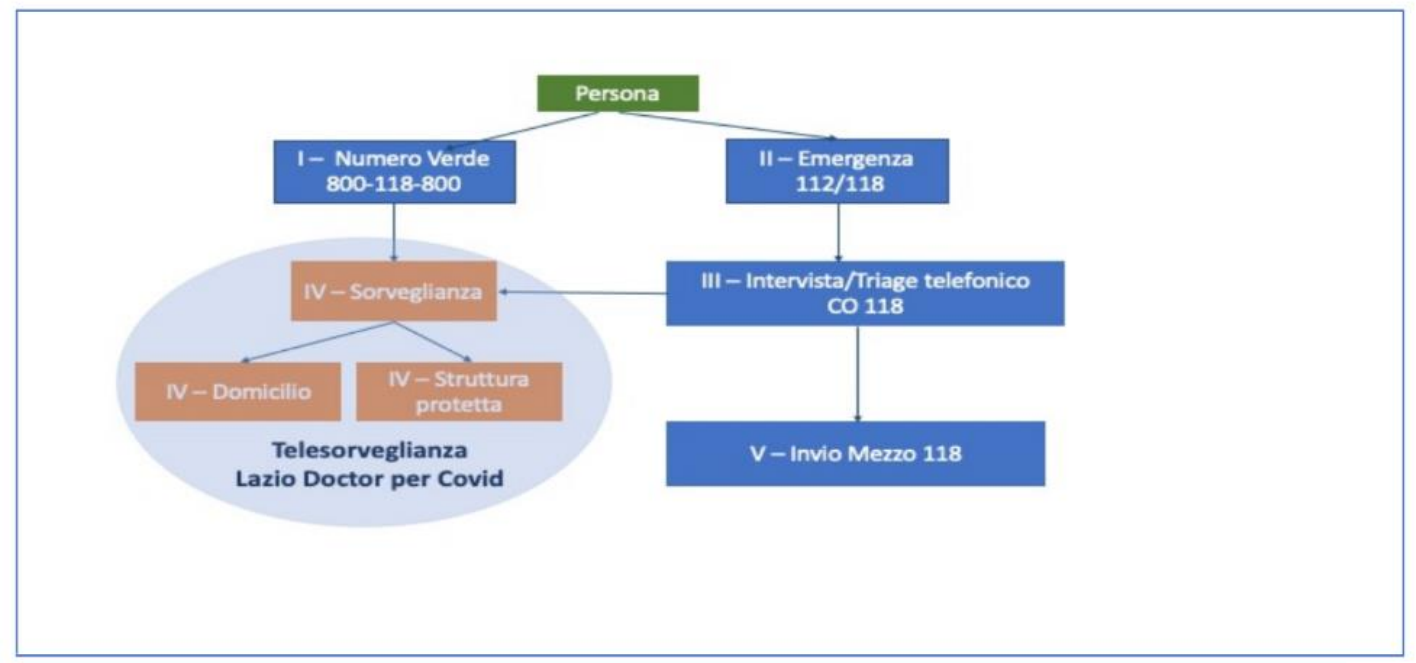

Fonte: Regione Lazio. Direzione Regionale Salute e Integrazione Sociosanitaria. Emergenza Covid-19 - Azioni di Fase II. Portaria 0237102 de 20/03/2020.

\section{II) FASE II - ESTRATÉGIAS PARA REORGANIZAR ATENÇÃO HOSPITALAR}

O aumento no número de casos registrado na Região de Lazio levou a implementação de medidas para reorganização da atenção hospitalares, as quais foram desencadeadas a partir do dia 20 de março de 2020. Dentre elas:

\section{II.1) Suspenção das atividades cirúrgicas}

A suspenção das atividades cirúrgicas foi implementada em hospitais públicos. Cirurgias de urgências e para tratamentos oncológicos, porém, foram mantidos em áreas definidas e bem separadas das alas destinadas ao atendimento de pacientes COVID. Essa medida, de acordo com a normativa do Ministério da Saúde, tem o duplo objetivo de concentrar os profissionais de saúde nas atividades Covid-19 e reduzir a presença desnecessária de pessoas nos hospitais ${ }^{3}$. 
II.2) Ativação progressiva de áreas de isolamento hospitalar

Áreas de isolamento fora do Pronto Socorro, normalmente usando tendas, foram progressivamente implementadas para acomodar casos em avaliação, ainda aguardando a resposta do teste diagnóstico, ou casos confirmados, pendentes de transferência. Essas áreas têm uma capacidade não inferior à 30 pessoas, número que vai ser modificado de acordo com a evolução epidemiológica.

\section{II.3) Ampliação progressiva de leitos}

A capacidade de leitos nos hospitais de referência para Covid-19 foi progressivamente ampliada. Quando não foi possível aumentar a capacidade do hospital de referência, a ampliação foi implementada em outras unidades da rede.

\section{II.4) Oxigenioterapia}

Instalações e equipamentos de oxigenioterapia foram instalados em todas as estações de trabalho das áreas hospitalares envolvidas na gestão de emergência, entendida como principal ferramenta de tratamento precoce.

\section{II.5) Equipe multidisciplinar de coordenação}

A constituição de equipes multidisciplinares de coordenação e referências clínicas representou uma medida implementada em cada hospital, com a seguinte composição: anestesiologista, enfermeiro, infectologista, internista, especialista em emergências, pneumologista. Essas equipes têm as seguintes tarefas:

O manter atualizadas as indicações clínicas do caso, protocolos e procedimentos;

- treinar operadores de diferentes disciplinas, que deverão estar envolvidos na gestão desses pacientes;

- apoiar os departamentos de doenças infecciosas e pneumologia para proporcionar cuidados sub-intensivos ou intensivos;

- garantir a cooperação em rede com os hospitais Covid-19.

\section{II.6) Transporte de pacientes}

O transporte de pacientes foi organizado de modo centralizado e coordenado pelo serviço de ambulâncias SAMU, que dispõe de veículos adequados e modalidades certificadas de higienização e cuidado de pacientes COVID-19.

\section{II.7) Regulação da ocupação de leitos}

A regulação da ocupação de leitos hospitalares é atribuição do gestor público com governança da totalidade dos leitos COVID-19, gerenciados pela Central de Regulação da Região Lazio.

\section{III) FASE III - ESTRATÉGIAS PARA REORGANIZAR LEITOS DE TERAPIA INTENSIVA}

A partir do 26 de março de $2020^{4}$, a evolução epidemiológica requereu a ativação da Fase III de resposta a situação de Emergência. Nesta fase buscou-se ampliar o número de leitos de unidades de terapia intensiva e de equipes multidisciplinares (infectologia, medicina Interna, pneumologia, reanimação) nos hospitais. A ampliação foi realizada na própria rede existente ou identificando novas instalações. As principais estratégias de previstas na fase III foram também dedicadas a fortalecer algumas ações das fases anterior, a saber:

- Expandir a áreas externas dos Prontos Socorros e Emergências dedicadas à espera dos resultados de diagnóstico de casos suspeitos;

- Melhorar o isolamento dos casos confirmados, no domicílio ou centros de acolhimento, com vigilância e monitoramentos remotos;

- Adaptar a capacidade de acolhimento hospitalar ao quadro epidemiológico, inclusive com instalações temporárias de saúde, de acordo com a normativa vigente;

- Diferenciar os fluxos de hospitalização em estruturas com diferentes intensidades de atendimento, a fim de concentrar os recursos para as unidades com alta intensidade de atendimento

- Aumentar os leitos com equipamentos de assistência respiratória e de terapia intensiva. 


\section{ANÁLISE DA EXPERIÊNCIA DA ITÁLIA E REFLEXÕES PARA O BRASIL}

A análise da experiência italiana apresenta importantes lições que podem auxiliar gestores de sistemas e serviços de saúde. Entretanto, é preciso considerar a realidade e contextos locais de implementação, em que experiências bem sucedidas podem não ser replicáveis ou ter efeitos diversos dos esperados, principalmente num país tão grande e diverso como o Brasil. Portanto, as lições servem muito mais como objeto de reflexão do que receitas a serem copiadas ao pé-da-letra.

Em primeiro lugar, a experiência italiana serve de alerta sobre riscos que as unidades de saúde, mantendo o seu funcionamento normal, sem tomar precauções extraordinárias, podem se tornar propagadores do vírus na população e coloquem em perigo a integridade física dos profissionais de saúde. Para enfrentar esse problema, uma das principais preocupações foi limitar o acesso presencial as unidades de saúde, visando minimizar o contato físico entre profissionais de saúde e usuários. Como alternativa, modalidades remotas de atendimento foram implementadas mediante uso massivo de instrumentos de telemedicina.

Ao analisar a realidade brasileira, verifica-se que uma parte da população, mesmo possuindo dispositivos móveis, tem escassa disponibilidade de acesso a conexões rápidas de Internet nos domicílios. Além disso, a pouca familiaridade de usuários e profissionais de saúde com teleconsulta e vigilância remota - em parte justificada pela histórica oposição da classe médica brasileira à introdução da telemedicina - pode dificultar a aplicação extensiva de essa modalidade no país.

Em segundo lugar, é importante destacar que as medidas de restrição do acesso espontâneo aos serviços de saúde aplicada na Itália foram acompanhadas por duas condições essenciais para evitar que isso representasse restrição real de acesso dos pacientes ao sistema de saúde. A primeira foi a organização de um sistema de transporte de pacientes, com rotinas de higienização certificadas e uma plataforma de operações tecnologicamente avançada e conectada online com a central de leitos da região. A segunda condição central para o modelo italiano foi fortalecer a governança de todos os leitos da região, incluindo os do setor privado, destinados a emergência, o que facilita a gestão eficiente dos recursos e dos fluxos. Em muitos lugares do Brasil estas condições parecem difíceis de alcançar, pelo menos nos tempos curtos que a emergência requer.

Em terceiro lugar, na Itália as medidas de distanciamento social e a vigilância dos positivos em isolamento domiciliar são muito estritas, com ações fiscalizadoras intensas, abrangentes e muito restritivas. No Brasil, existe uma enorme dificuldade de exercer uma vigilância efetiva em todo o território nacional. Além das dificuldades geográficas de acesso a lugares remotos, muitas regiões urbanas são afetadas por um contexto social violento, que inviabiliza ações efetivas de fiscalização no lugar.

Em quarto lugar, a resposta à epidemia de COVID-19 na Itália gerou um incremento gigantesco da demanda de alguns materiais médicos e equipamento essenciais, como oxigênio, EPIs e ventiladores. Atualmente, a demanda mundial por esses produtos extrapolou a capacidade de oferta, e certamente esse cenário não será diferente no o Brasil.

Por fim, uma questão potencialmente explosiva para o contexto brasileiro é o isolamento domiciliar, necessário nos casos positivos que não precisam de internação, além de outras circunstâncias. Pelas enormes desigualdades sociais, muitas famílias brasileiras moram em domicílios com condições que não permitam o isolamento de uma pessoa em condição de segurança. Isso vai requerer, possivelmente em tempos muito curtos, a instalação de centros de acolhimentos para essas pessoas, em ambientes adequados.

\section{CONCLUSÃO}

Concluímos esse artigo descritivo e reflexivo com um relato de autoria da equipe médica do 
Tasca, R.; Massuda, A.

Hospital de Bergamo, epicentro da epidemia na Itália. Neste artigo, a equipe de Bergamo descreve, de maneira clara e didática, o desmoronamento do sistema de saúde da Lombardia e avoca para um modelo de atenção projetado para o território e a comunidade, baseado na atenção primária à saúde:

\begin{abstract}
"Quanto mais medicalizada e centralizada a sociedade, mais disseminado o vírus. Essa catástrofe que se desenrola na rica Lombardia pode acontecer em qualquer lugar."... "Esse desastre poderia ser evitado apenas pela implantação maciça de serviços de atenção próximos às comunidades. Soluções contra a pandemia são necessárias para toda a população, não apenas para hospitais." (Mirco Nacoti et al., 2020) ${ }^{5}$
\end{abstract}


Tasca, R.; Massuda, A.

\section{NOTAS E REFERÊNCIAS}

1 Os autores agradecem os doutores Francesco Ripa di Mean (Diretor Geral, Istituti Fisioterapici Ospitalieri, Roma, Italia) e Martino Ardigò (Universidade de Bolonha, Itália), pelas contribuições ao artigo.

${ }^{2}$ Armocida B, Formenti B, Ussai S, Palestra F, Missoni E. The Italian health system and the COVID-19 challenge. The Lancet Public Health. 2020 Mar 25.

${ }^{3}$ Armocida B, Formenti B, Ussai S, Palestra F, Missoni E. The Italian health system and the COVID-19 challenge. The Lancet Public Health. 2020 Mar 25.

${ }^{4}$ Regione Lazio. Direzione Regionale Salute e Integrazione Sociosanitaria. Emergenza Covid-19 Fase III. Piano adottato in attuazione della circolare del Ministero della Salute prot. GAB. 2627. 26/03/2020.

${ }^{5}$ Mirco Nacoti et Al. At the Epicenter of the Covid-19 Pandemic and Humanitarian Crises in Italy: Changing Perspectives on Preparation and Mitigation. In a Bergamo hospital deeply strained by the Covid-19 pandemic, exhausted clinicians reflect on how to prepare for the next outbreak. Catalyst NEJM, 21 march. 